\title{
Low Thermal Conductivity in Nanocrystalline $\mathrm{Zn}_{3} \mathrm{P}_{2}$
}

\author{
W. D. Thompson, ${ }^{a^{*}}$ Rajesh Vaddi, ${ }^{b}$ B. E. White Jr. ${ }^{a, b}$ \\ ${ }^{a}$ Department of Physics, Binghamton University, 4400 Vestal Parkway East, \\ Binghamton, New York 13902, USA \\ ${ }^{\mathrm{b}}$ Materials Science and Engineering Program, Binghamton University, 4400 Vestal \\ Parkway East, Binghamton, New York 13902, USA
}

\begin{abstract}
Semiconductors with low lattice thermal conductivity are important in the search for more efficient thermoelectric materials. The thermal conductivity of nanocrystalline $(<7 \mathrm{~nm})$ $\mathrm{Zn}_{3} \mathrm{P}_{2}$, fabricated in thin film form by pulsed laser deposition, was measured from $80 \mathrm{~K}$ to $294 \mathrm{~K}$. The thermal conductivity of the film showed weak temperature dependence in this temperature range and at $294 \mathrm{~K}$ had its highest value of $0.49 \mathrm{~W} / \mathrm{m} \mathrm{K}$. Although $\mathrm{Zn}_{3} \mathrm{P}_{2}$ and its family of isomorphic compounds are known to have intrinsically low thermal conductivity, at room temperature the thermal conductivity of this nanocrystalline film is $25 \%$ smaller than the calculated minimum thermal conductivity for $\mathrm{Zn}_{3} \mathrm{P}_{2}$. Analyzing the thermal conductivity data with the Callaway model revealed that the data could be well fit by considering only boundary scattering and point defect scattering. The boundary scattering length was in good agreement with the film's average crystallite size of $4.1 \mathrm{~nm}$ and the magnitude of the point defect scattering required the formation of $\mathrm{V}_{\mathrm{Zn}}-\mathrm{Zn}_{\mathrm{i}}$ pairs from approximately $23 \%$ of the $\mathrm{Zn}$ sites. It is believed that a large number of point defects are responsible for the intrinsically low thermal conductivity of bulk $\mathrm{Zn}_{3} \mathrm{P}_{2}$ and therefore the exceptionally low thermal conductivity found in the present study results from the nanometer dimensions of the crystallites. As previous studies have reported high Seebeck coefficients and electronic properties that are insensitive to grain

${ }^{*}$ Corresponding Author at: Department of Physics, Binghamton University, 4400 Vestal Parkway East, Binghamton, New York 13902, USA Tel.:+16077772217. E-mail Address: wthomps1@binghamton.edu
\end{abstract}


boundaries in $\mathrm{Zn}_{3} \mathrm{P}_{2}$, the low thermal conductivity observed in the present study suggests that nanocrystalline $\mathrm{Zn}_{3} \mathrm{P}_{2}$ should be further explored for use in thermoelectric applications. Keywords: Thermal Conductivity; $\mathrm{Zn}_{3} \mathrm{P}_{2}$; Callaway Model; Nanostructured Materials; Point Defects

\section{Introduction}

The widespread implementation of thermoelectric type renewable energy sources would be bolstered by the development of efficient devices that are cost effective and formed from earth abundant materials. The efficiency of a thermoelectric is quantified by the dimensionless figure of merit, $Z T=S^{2} \sigma T /\left(\kappa_{e}+\kappa_{l}\right)$, where $\mathrm{S}$ is the Seebeck coefficient, $\sigma$ is electrical conductivity, $\kappa_{e}$ is the electronic contribution to the thermal conductivity and $\kappa_{l}$ is the thermal conductivity of the lattice. Optimization of ZT is made difficult by the interdependencies that exist between the electronic parameters. As a result, much of the focus when developing more efficient thermoelectrics has been placed on minimizing $\kappa_{l}$ and maximizing $S$. Large reductions of the lattice thermal conductivity have been achieved through nanostructuring (e.g. layered structures, nanowires and nanocrystalline solids ${ }^{1-4}$ ) and unit cell disorder (e.g. the insertion of "rattlers" in skutterudites and clathrates ${ }^{5-7}$ ). In systems with intrinsically low thermal conductivities, if care is taken to produce nanostructure dimensions that are smaller than the dominant phonon mean free paths of the bulk, the resulting thermal conductivities could be exceptionally low. ${ }^{8,9}$

Zinc-phosphide $\left(\mathrm{Zn}_{3} \mathrm{P}_{2}\right)$ is a type $\mathrm{A}_{3} \mathrm{II}_{3} \mathrm{~V}_{2}$ semiconductor with a direct optical bandgap of $1.5 \mathrm{eV} .{ }^{10}$ It is composed entirely of earth abundant elements and has low production costs. ${ }^{11}$ The $\mathrm{Zn}_{3} \mathrm{P}_{2}$ unit cell, shown in Fig. 1, contains 40 atoms and its crystal structure is described as that of a distorted anti-flourite structure, where only three quarters of the voids formed by the 
phosphorus tetrahedra are occupied by zinc atoms, leaving the remaining voids vacant. ${ }^{12}$ As an example, in Fig. 1 four such vacant voids are highlighted in green. Studies on $\mathrm{Zn}_{3} \mathrm{P}_{2}$ and its family of isomorphic compounds $\left(\mathrm{Cd}_{3} \mathrm{P}_{2}, \mathrm{Zn}_{3} \mathrm{As}_{2}\right.$, and $\left.\mathrm{Cd}_{3} \mathrm{As}_{2}\right)$ have revealed low lattice thermal conductivity as an intrinsic property of the distorted anti-flourite structure. ${ }^{13-15}$ The lowered symmetry of the crystal structure, the resulting distribution of bond lengths, both covalent (56 of the 96 bonds) and ionic (40 of the 96 bonds) in nature, and the large unit cell are all properties shown to correlate with low thermal conductivity materials. ${ }^{16-19}$ Brockway et al. ${ }^{20}$ reported room temperature thermal conductivities of $\mathrm{Zn}_{3} \mathrm{P}_{2}$ nanowire (30-50 nm diameter) pellets which were $14 \%$ higher than the thermal conductivity of $\mathrm{Zn}_{3} \mathrm{P}_{2}$ samples measured by Nagamoto et al. ${ }^{21}$, having much larger crystallites $(\sim 25 \mu \mathrm{m})$. These findings are contrary to the expectation that increased phonon scattering at the boundaries of the nanostructures should result in a reduction in thermal conductivity. Although there have been numerous reports on the optoelectronic properties of this compound ${ }^{10,12,22-24}$, stemming from interest for its incorporation in photovoltaic (PV) devices, there are relatively few studies which report on the thermal properties. With previous studies having reported large Seebeck coefficients ${ }^{15,20,21,25}(1.8 \mathrm{mV} / \mathrm{K}$ at room temperature reported by Babu et al. ${ }^{25}$ ) and no appreciable changes to the electrical conductivity in the presence of grain boundaries ${ }^{22,25}$, further reductions of the lattice thermal conductivity through nanostructuring could be beneficial to increasing the viability of this compound for use in thermoelectric applications. In this work the thermal conductivity of $\mathrm{Zn}_{3} \mathrm{P}_{2}$ thin films with crystallite sizes less than $10 \mathrm{~nm}$ is presented. 


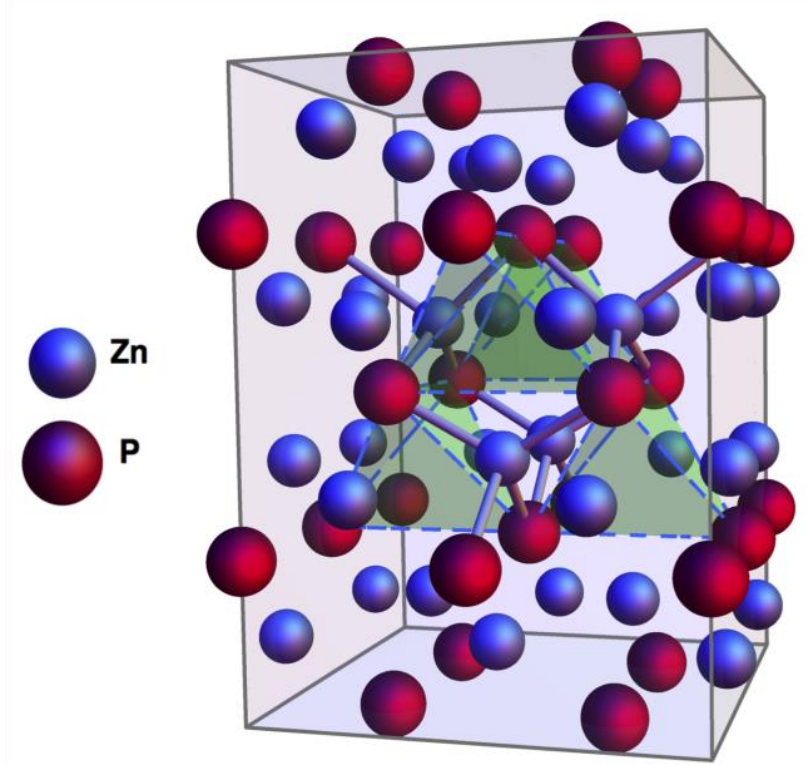

Fig. 1. The $\mathrm{Zn}_{3} \mathrm{P}_{2}$ unit cell. The 40 atom unit cell is tetragonal, with phosphorus and zinc planes alternating along the c-axis. The crystal structure is described as a distorted anti-flourite structure where only three quarters of the tetrahedral voids formed by phosphorus atoms are occupied by zinc atoms. The green tetrahedra highlight several of the tetrahedral voids that are vacant in the unit cell.

\section{Experimental Details}

\subsection{Sample Fabrication}

Using a pulsed laser deposition (PLD) system, a $500 \mathrm{~nm} \mathrm{Zn}_{3} \mathrm{P}_{2}$ thin film was deposited onto a silicon wafer on which $100 \mathrm{~nm}$ of thermally grown $\mathrm{SiO}_{2}\left(t h-\mathrm{SiO}_{2}\right)$ had previously been deposited. The PLD system used a KrF excimer laser (Neocera, lambda=248 nm, pulse width=25 ns) with a pulse repetition rate of $10 \mathrm{~Hz}$. For a stoichiometric film, deposition was carried out at room temperature with a pressure of $1.2 \times 10^{-6}$ Torr using a $99.9 \% \mathrm{Zn}_{3} \mathrm{P}_{2}$ target (Plasmaterials), a laser energy density of $3 \mathrm{~J} / \mathrm{cm}^{2}$, and a substrate to target distance of $50 \mathrm{~cm}$. Post-deposition processing of the sample included rapid thermal annealing (RTA) in a nitrogen ambient, where 
the temperature was increased at $150 \mathrm{~K} / \mathrm{s}$, held at $773 \mathrm{~K}$ for $120 \mathrm{~s}$ and then decreased to room temperature at $20 \mathrm{~K} / \mathrm{s}$. Annealing at higher temperature or for longer durations resulted in the sample deviating from stoichiometry.

\subsection{Structural Characterization}

$\mathrm{X}$-ray diffraction (XRD) data for the film was collected at room temperature using a PANalytical X'Pert Pro Materials Research Diffractometer with a Cu-Ka source $\left(\lambda=1.54 \times 10^{-10}\right.$ $\mathrm{m})$ in the grazing incidence configuration. The resulting XRD pattern was used for phase identification, determination of the lattice constants and, with subsequent Scherrer Analysis, determination of the crystallite sizes of the film. The crystallite sizes and morphologies were observed using a high resolution transmission electron microscopy (HRTEM) system (JEM 2100F from JEOL).

\subsection{Thermal Conductivity Measurement}

Thermal conductivity measurements were made using the $3 \omega$-method popularized by Cahill for measurements on bulk solids and later extended to measurements of thin films. ${ }^{26,29}$ In this work the heater line was fabricated by electron beam evaporation of a $3 \mathrm{~nm}$ chromium adhesion layer and a $50 \mathrm{~nm}$ gold layer, followed by subsequent photolithographic patterning to the desired heater line dimensions of $1000 \mu \mathrm{m}$ x $20 \mu \mathrm{m}$. The electrical conductivity of the $\mathrm{Zn}_{3} \mathrm{P}_{2}$ film was large enough to cause spurious $3 \omega$ signals. It was necessary to deposit an electrically insulating film between the $\mathrm{Zn}_{3} \mathrm{P}_{2}$ sample and the metal line to prevent this. Using an AJA Orion sputter deposition system, a $180 \mathrm{~nm}$ amorphous $\mathrm{SiO}_{2}\left(a-\mathrm{SiO}_{2}\right)$ film was deposited to serve as the

electrical insulation between the $\mathrm{Zn}_{3} \mathrm{P}_{2}$ film and metal line. As the effect of multiple thin films on a substrate are additive, the thermal conductivity of this $a-\mathrm{SiO}_{2}$ film, as well as that of the th- 
$\mathrm{SiO}_{2}$ film, were measured independently in order to later subtract their contributions to the overall measurement. The thermal conductivity and thermal diffusivity of single crystal silicon from Ref. 30 were used to calculate the thermal response of the substrate.

\section{Results}

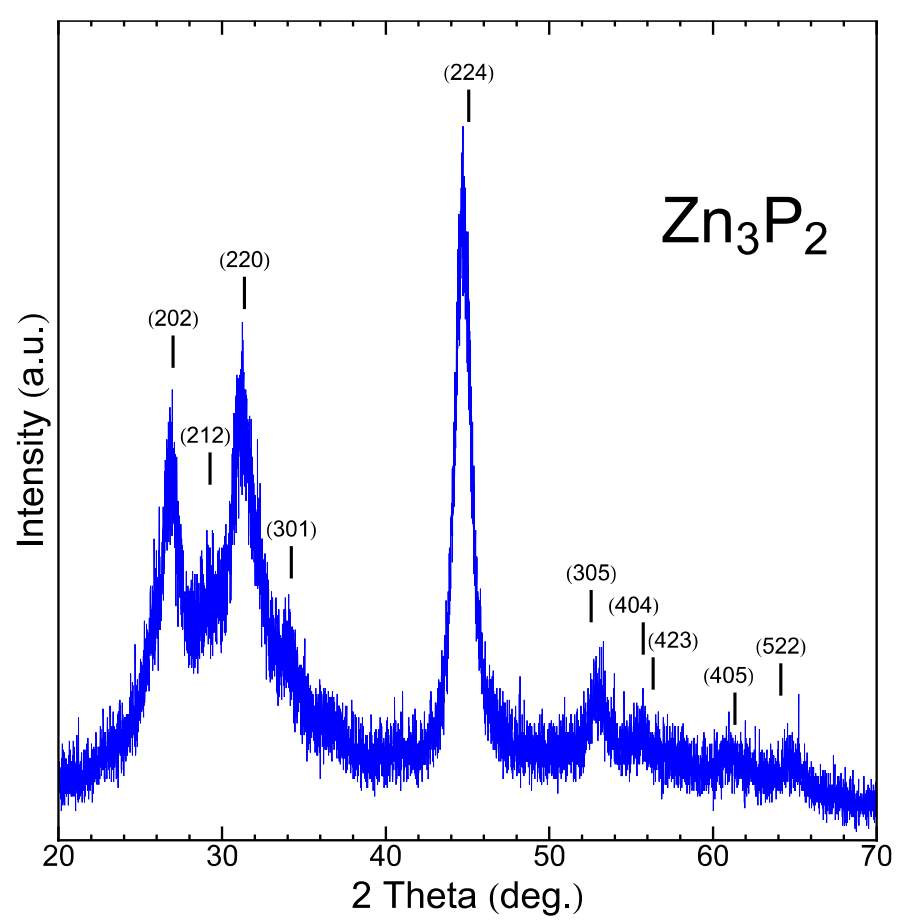

Fig. 2. The XRD profile for $\mathrm{Zn}_{3} \mathrm{P}_{2}$. The angle used for the grazing incidence scan was $\omega=0.60^{\circ}$. The sample is polycrystalline and displays the characteristic (202), (220), and (224) peaks of tetragonal $\mathrm{Zn}_{3} \mathrm{P}_{2}$, along with other minor $\mathrm{Zn}_{3} \mathrm{P}_{2}$ peaks.

\subsection{Structural Characterization}

The XRD pattern shown in Fig. 2, where an incident angle of $\omega=0.60^{\circ}$ was used, indicates that the sample is polycrystalline with no preferential orientation, displaying the characteristic (202), (220), and (224) peaks of tetragonal $\mathrm{Zn}_{3} \mathrm{P}_{2}$ along with other minor $\mathrm{Zn}_{3} \mathrm{P}_{2}$ peaks (JCPDS Card: 01-073-4212). From the location of the (202), (220), (224), and (305) 
peaks, the average unit cell dimensions are calculated as $\mathrm{a}=\mathrm{b}=8.10 \AA$ and $\mathrm{c}=11.43 \AA$. The accepted values for the $\mathrm{Zn}_{3} \mathrm{P}_{2}$ unit cell are $\mathrm{a}=\mathrm{b}=8.09 \AA$ and $\mathrm{c}=11.41 \AA .{ }^{31}$ Using a PseudoVoigt model fitting function, the (202), (220), and (224) peaks were fit and the crystallite sizes were determined according to the Scherrer equation ${ }^{32}$ :

$$
\mathrm{L}=\frac{K \lambda}{B \cos (\theta)}
$$

where $\mathrm{L}$ is the crystallite size, $\mathrm{K}$ is the Scherrer constant (taken as $0.94{ }^{33}$ ), $\mathrm{B}$ is the peak width in radians and $2 \theta$ is the location of the peak maximum. The fittings resulted in average crystallite sizes of $3.0 \mathrm{~nm}, 2.9 \mathrm{~nm}$, and $6.3 \mathrm{~nm}$ for the (202), (220), and (224) peaks, respectively, giving an overall average crystallite size of $4.1 \mathrm{~nm}$. The HRTEM image in Fig. 3 also indicates the existence of multiple crystallites with sizes in the $<10 \mathrm{~nm}$ regime and varying crystallographic orientations in the $\mathrm{Zn}_{3} \mathrm{P}_{2}$ film, in good agreement with the XRD data.

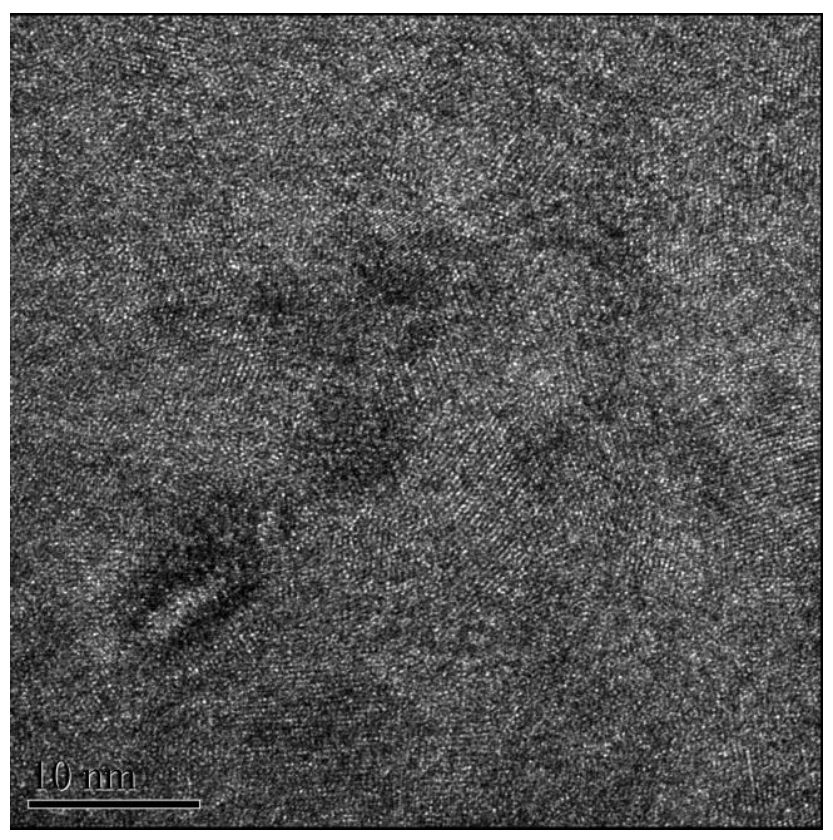

Fig. 3. The HRTEM image of the $\mathrm{Zn}_{3} \mathrm{P}_{2}$ film. Many crystallites with a variety of interplanar spacings can be seen, having dimensions that are found to be in good agreement with sizes determined from the analysis of the XRD peaks. 


\subsection{Thermal Conductivity}

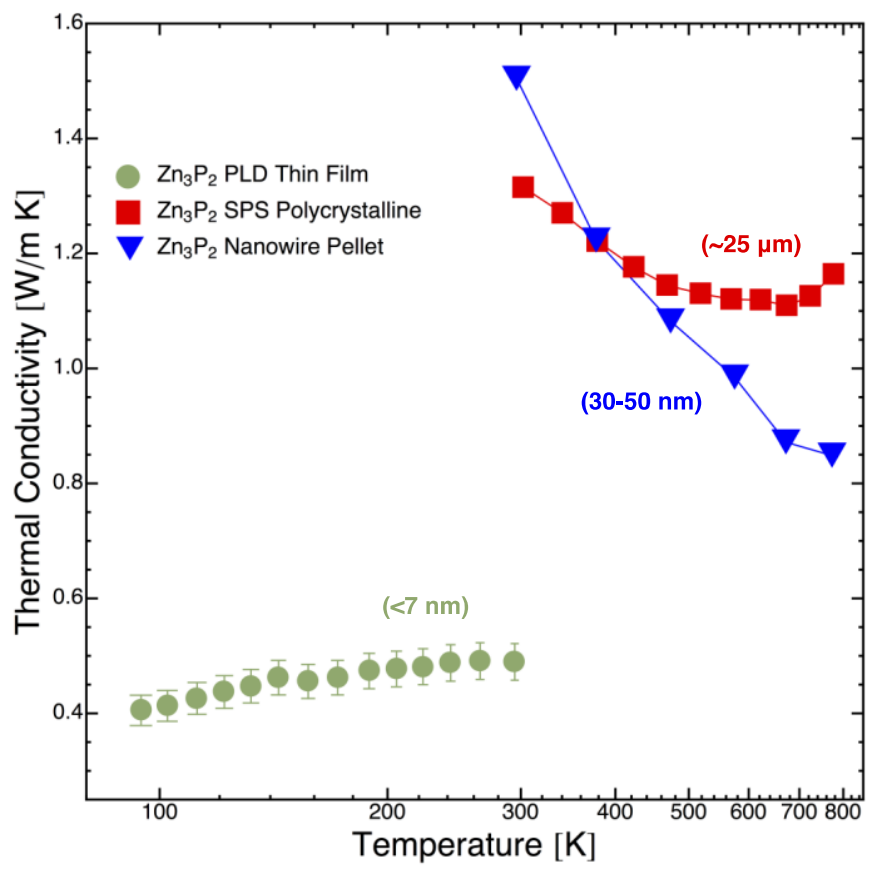

Fig. 4. The thermal conductivity data for $\mathrm{Zn}_{3} \mathrm{P}_{2}$. The thermal conductivity data for the $\mathrm{Zn}_{3} \mathrm{P}_{2} \mathrm{PLD}$ thin film of the present study, measured from $80 \mathrm{~K}$ to room temperature, are shown as green circles. The red squares are thermal conductivity data taken from Nagamoto et al. ${ }^{21}$ for samples of SPS polycrystalline $\mathrm{Zn}_{3} \mathrm{P}_{2}$. The blue inverted triangles are thermal conductivity data taken from Brockway et al. ${ }^{20}$ for $\mathrm{Zn}_{3} \mathrm{P}_{2}$ nanowire (30-50 $\mathrm{nm}$ in diameter) pellets, consolidated through hot uniaxial pressing.

In Fig. 4, the temperature dependent thermal conductivity data of the PLD $\mathrm{Zn}_{3} \mathrm{P}_{2}$ thin film are shown as green circles. The overall uncertainty in the reported data, more than half of which comes from the uncertainty in the measurement of the film thickness, is indicated by the error bars. To the authors' knowledge this is the first reported data on the thermal conductivity of $\mathrm{Zn}_{3} \mathrm{P}_{2}$ thin films. The thermal conductivity of the $\mathrm{Zn}_{3} \mathrm{P}_{2}$ film shows weak temperature 
dependence in the temperature range measured. The thermal conductivity of $0.49 \mathrm{~W} / \mathrm{m} \mathrm{K}$ at 294 $\mathrm{K}$ is the maximum value observed in the temperature range measured. For comparison, also shown in Fig. 4 are data from other reports on the thermal conductivity of $\mathrm{Zn}_{3} \mathrm{P}_{2}$. Nagamoto et $a l .{ }^{21}$ measured the thermal conductivity of SPS polycrystalline $\mathrm{Zn}_{3} \mathrm{P}_{2}$ samples, which were fabricated from solid-state reacted powders, shown as red squares in Fig. 4. Brockway et al. ${ }^{20}$ used hot uniaxial pressing to consolidate $\mathrm{Zn}_{3} \mathrm{P}_{2}$ nanowires synthesized through reactive vapor transport of red phosphorus onto zinc foils, shown as blue inverted triangles in Fig. 4.

\section{Discussion}

In Fig. 4, it can be seen that at room temperature the $\mathrm{Zn}_{3} \mathrm{P}_{2}$ thin film sample measured in this work falls a factor of 3 below that of the $\mathrm{Zn}_{3} \mathrm{P}_{2}$ SPS powders and $\mathrm{Zn}_{3} \mathrm{P}_{2}$ nanowire pellet. It is expected that a main reason for the observed reduction in thermal conductivity between the $\mathrm{Zn}_{3} \mathrm{P}_{2}$ film and the other samples results from increased boundary scattering, where for instance the nanowires measured by Brockway et al. ${ }^{20}$ were $30-50 \mathrm{~nm}$ in diameter as compared to the 2.9$6.3 \mathrm{~nm}$ crystallite sizes of the current study. Although the crystallite sizes for the SPS $\mathrm{Zn}_{3} \mathrm{P}_{2}$ powders measured by Nagamoto et al. ${ }^{21}$ were unavailable, during fabrication the powders were passed through a 500 mesh sieve ( $25 \mu \mathrm{m}$ openings) before SPS at high temperature, suggesting the presence of crystallites which are at least $25 \mu \mathrm{m}$ in diameter. As previously mentioned, it is peculiar that the thermal conductivity of the $\mathrm{Zn}_{3} \mathrm{P}_{2}$ nanowire pellet is found above that of the SPS microcrystals, where the electronic component of the thermal conductivity was negligible in both studies, while (as it will be shown) nanostructuring produces significant reductions in the thermal conductivity of the $\mathrm{Zn}_{3} \mathrm{P}_{2}$ film. Also of interest is the weak temperature dependence observed in the thermal conductivity of the $\mathrm{Zn}_{3} \mathrm{P}_{2}$ film. A temperature independent thermal conductivity is 
typically observed in glasses and is known to occur in grossly disordered crystalline solids, for example with large concentrations of point defects. ${ }^{34}$ Given that the XRD pattern in Fig. 2 clearly indicates that the film is crystalline, the latter seems to be a better explanation.

\subsection{Callaway Model}

To further explore the thermal conduction limiting mechanisms in the $\mathrm{Zn}_{3} \mathrm{P}_{2}$ thin film, the thermal conductivity data were fit using the Callaway model with considerations of boundary $\left(\tau_{\mathrm{B}}\right)$, point defect $\left(\tau_{\mathrm{PD}}\right)$, umklapp $\left(\tau_{\mathrm{U}}\right)$, and normal $\left(\tau_{\mathrm{N}}\right)$ scattering processes, where $\tau_{\mathrm{i}}$ are the associated relaxation times for each process. ${ }^{35}$ From the Wiedemann-Franz law and the room temperature electrical conductivity of $128(\Omega \mathrm{m})^{-1}$ (as determined from the charge carrier concentration of $2 \times 10^{18} \mathrm{~cm}^{-3}$ and charge carrier mobility of $4 \mathrm{~cm}^{2} / V \cdot s$ ), the electronic contribution to the thermal conductivity is found to be negligible and the total thermal conductivity is given almost entirely by the lattice component. Callaway gives the thermal conductivity of the lattice as

$$
\kappa_{L}=\frac{k_{B}}{2 \pi^{2} v}\left(\frac{k_{B} T}{\hbar}\right)^{3} \int_{0}^{\Theta_{D} / T} \tau_{C} \frac{x^{4} e^{x}}{\left(e^{x}-1\right)^{2}} d x
$$

where $x=\left(\hbar \omega / k_{B} T\right)$ is a dimensionless variable, $\omega$ is the phonon frequency, $k_{B}$ is the Boltzmann constant, $\hbar$ is the reduced Planck constant, $v$ is the average sound velocity, $\Theta_{D}=$ $v\left(\hbar / k_{B}\right)\left(6 \pi^{2} n\right)^{1 / 3}$ is the Debye temperature, $n=5.31 \times 10^{28} \mathrm{~m}^{-3}$ is the atomic number density, and $\tau_{C}$ is the combined relaxation time. From Ref. 36 the longitudinal and transverse sound velocities for $\mathrm{Zn}_{3} \mathrm{P}_{2}$ are $v_{l}=5290 \mathrm{~m} / \mathrm{s}$ and $v_{t}=2000 \mathrm{~m} / \mathrm{s}$, respectively, from which the average sound velocity and Debye temperature are calculated as $2269 \mathrm{~m} / \mathrm{s}$ and $254 \mathrm{~K}$, respectively. Matthiessen's rule for addition of the relaxation times gives the combined relaxation rate, $\tau_{C}^{-1}$, as 


$$
\begin{aligned}
\tau_{C}^{-1} & =\tau_{B}^{-1}+\tau_{P D}^{-1}+\tau_{U}^{-1}+\tau_{N}^{-1} \\
& =v / L_{C}+A \omega^{4}+B 1 \omega^{2} T e^{-\Theta_{D} / T}+B 2 \omega^{2} T
\end{aligned}
$$

where $L_{C}$ is the Casimir length and A, B1, and B2, are the coefficients for point defect, umklapp and normal scattering processes, respectively.

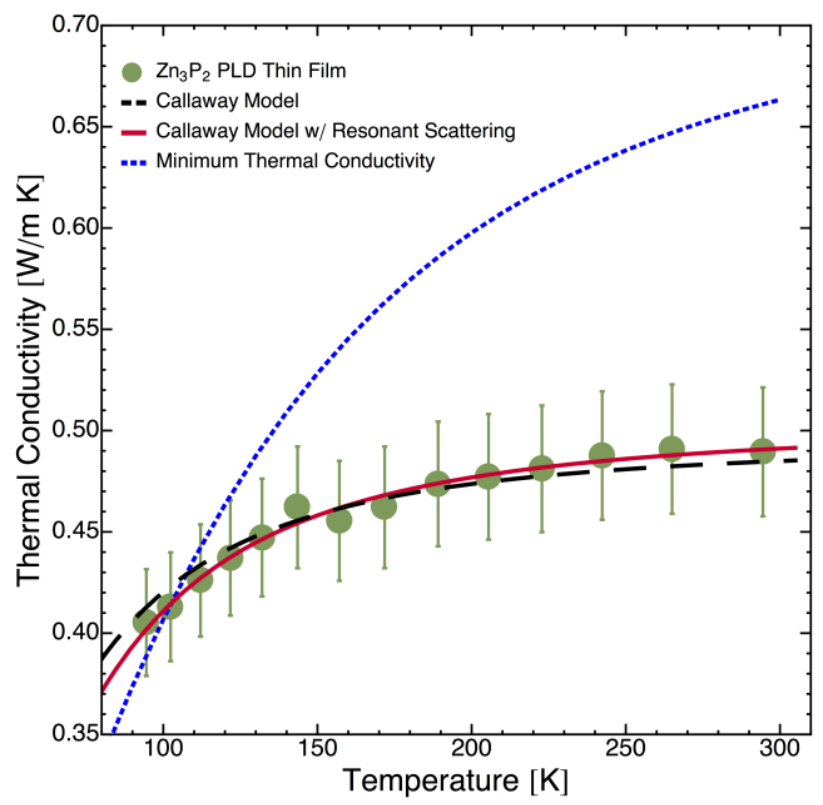

Fig. 5. Thermal conductivity models. The thermal conductivity data for the $\mathrm{Zn}_{3} \mathrm{P}_{2}$ thin film are shown as green circles. The best fit to the data found using the Callaway model with considerations of boundary, point defect, umklapp, and normal phonon scattering processes is shown as a black line with large dashing. The Callaway model was made to also include the effect of resonant phonon scattering processes and the resulting best fit to the data is shown as a solid red line. The minimum thermal conductivity for $\mathrm{Zn}_{3} \mathrm{P}_{2}$ is also shown, and is given as a blue line with small dashing. 
The fitting parameters that resulted in the best fit to the data, as determined through the minimization of $\chi^{2}$, were: $\mathrm{LC}_{\mathrm{C}}>2.0 \mathrm{~nm}, \mathrm{~A} \rightarrow 3.2 \times 10^{-41} \mathrm{~s}^{3}, \mathrm{~B} 1 \rightarrow 0$ and B2 $\rightarrow>0$, for which the resulting fit is shown in Fig. 5 as a dashed black line. The fit achieved overlays well with the thermal conductivity data of the $\mathrm{Zn}_{3} \mathrm{P}_{2}$ film, shown in green circles, and it is interesting to note that the inclusion of both phonon-phonon scattering processes, normal and umklapp, was unnecessary to fit the data in the temperature range considered. The Casimir length, which generally has the dimensions of the film crystallite size ${ }^{37}$, determined from the fitting is in good agreement with the average crystallite size of $4.1 \mathrm{~nm}$ determined from Scherrer analysis. It is then interesting to consider the phonon scattering that arises from point defects.

\subsubsection{Point Defect Phonon Scattering}

If boundary scattering were the only phonon scattering process included in the Callaway model, the thermal conductivity would become a factor of 4.5 larger at room temperature, indicating the significant reduction in thermal conductivity that results from the presence of point defects. As measured by Energy Dispersive X-ray analysis (EDX), the $\mathrm{Zn}_{3} \mathrm{P}_{2}$ film is stoichiometric. It is therefore difficult to believe that there exists a significant number of vacancies or interstitials associated with a deficiency or excess of constituent elements, respectively. It is also possible that there are instead antisite defect pairs, being $\mathrm{Zn}_{\mathrm{P}}$ and $\mathrm{P}_{\mathrm{Zn}}$, but

Demers et al. ${ }^{38}$ determined that such defects are too unstable and would result in the formation of $\mathrm{Zn}_{\mathrm{i}}-\mathrm{V}_{\mathrm{P}}$ and $\mathrm{P}_{\mathrm{i}}-\mathrm{V}_{\mathrm{Zn}}$, respectively. Given the atomic density of the unit cell, the space needed for even a moderate number of defect pairs of this type is also unavailable. Furthermore, the lattice constants calculated using the XRD data for the $\mathrm{Zn}_{3} \mathrm{P}_{2}$ film were in good agreement with the accepted values, indicating the absence of large distortions to the unit cell. The most 
energetically favorable defect types in $\mathrm{Zn}_{3} \mathrm{P}_{2}$ are that of phosphorus interstitials, $\mathrm{P}_{\mathrm{i}}$, and the less energetically favorable zinc vacancy, $\mathrm{VZn}^{39}{ }^{39}$ As there are considerably more sites ( 8 tetrahedral voids per unit cell) in which a $\mathrm{Zn}_{\mathrm{i}}$ can reside (necessary for accommodating a $\mathrm{V} Z$ n given that the sample is stoichiometric), as compared to the lone suitable site per unit cell for accommodating a $\mathrm{P}_{\mathrm{i}}{ }^{39}$, the point defect scattering that results from the formation of a $\mathrm{V}_{\mathrm{Zn}}-\mathrm{Zn}_{\mathrm{i}}$ pair is considered.

The point defect scattering parameter was given by Klemens ${ }^{40}$ as

$$
\mathrm{A}=\frac{V_{0} \Gamma}{4 \pi v^{3}}
$$

where $V_{0}$ is the volume per atom, $v$ is the average velocity, and $\Gamma$ is the disorder scattering parameter. $\Gamma$ is given by the sum of two terms which quantify the effects of mass $\operatorname{disorder}\left(\Gamma_{M}\right)$ and strain field $\left(\Gamma_{S}\right)$ scattering that result from a point defect. However, because of the uncertainty associated with determining the effective radii of these defects, which is required for calculation of the strain field scattering, only mass disorder scattering will be considered in the following. Furthermore, from the density functional theory (DFT) work of Demers and van de Walle ${ }^{38}$ on the defect types in $\mathrm{Zn}_{3} \mathrm{P}_{2}$, there is reason to believe that the effective radii difference between a $\mathrm{Zn}$ ion and $\mathrm{V}_{\mathrm{Zn}}$ in $\mathrm{Zn}_{3} \mathrm{P}_{2}$ may be less than $5 \%$, which would result in a negligibly small $\Gamma_{S}$ term. Slack ${ }^{41}$ developed an expression for the mass disorder scattering parameter for a compound, which in the case of a binary compound of the form $\mathrm{A}_{\mathrm{x}} \mathrm{B}_{\mathrm{y}}$ is given as

$$
\Gamma_{M}\left(A_{x} B_{y}\right)=\frac{x}{x+y}\left(\frac{\bar{M}_{A}}{\bar{M}}\right)^{2} \Gamma_{M}(A)+\frac{y}{x+y}\left(\frac{\bar{M}_{B}}{\bar{M}}\right)^{2} \Gamma_{M}(B)
$$

where $\bar{M}_{i}$ are the average masses at the $i$ th $(i=A, B)$ site in the unit cell, and $\bar{M}$ is the average mass of all atoms in the unit cell. The $\Gamma_{M}(i)$ are the scattering coefficients associated with the mass disorder present at the $i$ th site and are given by 


$$
\Gamma_{M}(i)=\sum_{j} f_{j} \frac{\left(\mathrm{M}_{j}-\bar{M}_{i}\right)^{2}}{\left(\bar{M}_{i}\right)^{2}}
$$

where $f_{j}$ are the fractional concentrations of defects with mass $M_{j}$ at the $i$ th site and $\bar{M}_{i}$ is the average mass of the $i$ th site. If for example there is no disorder on site $\mathrm{B}, \Gamma(B)=0$.

From (6) it is clear that the zinc vacancy is the single most effective mass disorder type point defect in $\mathrm{Zn}_{3} \mathrm{P}_{2}$, as it represents the largest mass difference. Considering only this defect type, with (5) and (6) it is found that $23 \%$ of the $\mathrm{Zn}$ lattice sites would necessarily be occupied by vacancies to achieve the strength of point defect scattering found from fitting to the Callaway model. The reader is reminded that this result represents an upper limit to the magnitude of $\Gamma_{M}$, as the $\Gamma_{S}$ associated with these $\mathrm{Zn}$ vacancies and possible disorder on $\mathrm{P}$ sites may also contribute to the total point defect scattering.

\subsubsection{Resonant Phonon Scattering}

It is expected that in the stoichiometric $\mathrm{Zn}_{3} \mathrm{P}_{2}$ film a large number of $\mathrm{V} \mathrm{Zn}$ defects should be accompanied by a proportionate number $\mathrm{Zn}_{\mathrm{i}}$ defects. Furthermore, it is known that interstitial type defects will have associated with them an additional mechanism by which phonons can be scattered in a solid. ${ }^{42}$ Most notably, in the case of filled clathrates and filled skutterudites a "guest" atom or molecule is loosely bound and free to oscillate (or "rattle") within a void created by the host structure, and can lead to a marked reduction in thermal conductivity. ${ }^{6,43-45}$ The modes associated with these "rattlers" are highly localized and as a result have been modeled as Einstein oscillators. ${ }^{46,47}$ A resonant type interaction of the "rattlers" with the low frequency normal modes of the solids has, with great success, been modeled using the resonant phonon scattering formalism put forth by Pohl. ${ }^{7,48-51}$ Pohl provides an additional resonant scattering term to be included in the total phonon relaxation rate, given as 


$$
\tau_{r e s}^{-1}=\frac{\mathrm{C} \omega^{2}}{\left(\omega_{0}^{2}-\omega^{2}\right)^{2}}
$$

where $\omega$ is the phonon frequency, $\mathrm{C}$ is a fitting parameter that is proportional to the number of resonant scatterers, and $\omega_{0}$ is the resonant frequency.

The total relaxation rate of (3) was made to include the additional resonant scattering term given by (7) and the data were refit, however having the boundary scattering term fixed at its previously determined value. The resulting fitting parameters are: $\mathrm{L}_{\mathrm{C}} \rightarrow>2.0 \mathrm{~nm}, \mathrm{~A}->2.0 \mathrm{x}$ $10^{-41} \mathrm{~s}^{3}, \mathrm{~B} 1->0, \mathrm{~B} 2->0, \mathrm{C}->2.8 \times 10^{37} \mathrm{~s}^{-3}$ and $\omega_{0->} 1.4 \times 10^{13} \mathrm{rad} / \mathrm{s}$ and the resulting fit is shown in Fig. 5 as a solid red line. With the inclusion of the resonant scattering term, a slightly better fit is achieved in the low and high temperature ranges of the thermal conductivity data. A significant feature of this result is that the point defect scattering term remains close to its original value, even with the inclusion of the resonant scattering term. The previous analysis for point defect scattering is still applicable and it is encouraging that the data is well described by the fit after accounting for the presence of interstitial atoms, albeit whose effect on the thermal conductivity is small in the temperature range measured. Klitsner and Pohl ${ }^{52}$ have used

$$
v_{d o m}=\left(90 \frac{G H z}{K}\right) T
$$

to approximate the frequency of the phonons that dominate thermal transport at a given temperature, where $v_{d o m}$ is the phonon frequency in $\mathrm{Hz}$. For the $2.2 \mathrm{THz}$ resonant frequency determined from the Callaway model fitting, (8) suggests that the effect of resonant scattering would be greatest near $24 \mathrm{~K}$. Therefore an extension of the thermal conductivity measurements to much lower temperatures would be necessary to gain additional insight about the resonant scattering in this system. 


\subsubsection{The Origin of High Concentrations of Point Defects}

As it draws many similarities to the considerations of the present study we mention briefly the semiconducting compound $\beta-\mathrm{Zn}_{4} \mathrm{Sb}_{3} . \beta-\mathrm{Zn}_{4} \mathrm{Sb}_{3}$ is in a class of materials known as "phonon-glass electron-crystals" 53 and has one of the highest thermoelectric figures of merit, ZT, in the temperature range of 473-673 K, owing to its exceptionally low lattice thermal conductivity (Room temperature values from $0.65 \mathrm{~W} / \mathrm{m} \mathrm{K}$ for bulk to $0.10 \mathrm{~W} / \mathrm{m} \mathrm{K}$ for thin films have been reported. $)^{54-56}$ Synchrotron powder diffraction resolved electron density analysis and DFT calculations have established that this low lattice thermal conductivity results from as many as $20 \%$ of the $\mathrm{Zn}$ atoms sitting on three distinct interstitial sites with a large percentage of the 36 $\mathrm{Zn}$ lattice sites being left vacant. ${ }^{9,55,57,58}$ The $\mathrm{Zn}$ vacancies provide highly effective centers for point defect scattering and the three unique interstitial sites should have associated with them three unique resonant frequencies that are able to resonantly scatter long wavelength phonons. A study of the temperature dependent occupancy of the $\mathrm{Zn}$ lattice site and the $\mathrm{Zn}_{\mathrm{i}}$ site revealed that occupancy of the $\mathrm{Zni}$ site was highly favored at high temperature. Furthermore, it has been shown that rapid cooling after the annealing of $\beta-\mathrm{Zn}_{4} \mathrm{Sb}_{3}$ prevented the room temperature equilibrium population of the $\mathrm{Zn}$ lattice site from being established. ${ }^{59}$ This is consistent with other reports of rapid cooling after high temperature annealing during the fabrication of $\beta-\mathrm{Zn}_{4} \mathrm{Sb}_{3}$ samples in which low thermal conductivity was reported. ${ }^{54}$

In an XRD study coupled with structure refinement software, Zanin et al. ${ }^{60}$ have pointed out that of the four tetrahedra in $\mathrm{Zn}_{3} \mathrm{P}_{2}$, two are distinctly occupied by two of three distinct $\mathrm{Zn}$ atoms of the lattice. Furthermore, there is an equal probability for the remaining $\mathrm{Zn}$ atom, known as the $\mathrm{Zn}(2)$ atom, to reside in either of 2 remaining voids in the lattice leaving the other as a vacant void. Figure 1 helps to illustrate this idea where the $\operatorname{Zn}(2)$ atom is shown being 
bonded tetrahedrally to the surrounding P atoms, and the adjacent void sites, highlighted by the green tetrahedra, are the equivalent positions in which the $\mathrm{Zn}(2)$ atom can reside. Although Zanin et al. ${ }^{60}$ state that in equilibrium only one of these two sites is continuously occupied throughout the crystal by the $\mathrm{Zn}(2)$ atom, their observation comes as a result of the structure refinement software producing an equilibrium state in the $\mathrm{Zn}_{3} \mathrm{P}_{2}$ system. In the present study, the RTA process subjected the $\mathrm{Zn}_{3} \mathrm{P}_{2}$ film to annealing at $773 \mathrm{~K}$ for $120 \mathrm{~s}$, followed by rapid cooling at $20 \mathrm{~K} / \mathrm{s}$ to room temperature. It is likely that the RTA process disturbs the equilibrium configurations of the $\mathrm{Zn}(2)$ and vacancy void sites and with the rapid cooling that follows, reestablishment of the equilibrium populations of these two sites is not possible. This would then result in a large number of $\mathrm{V}_{\mathrm{Zn}}-\mathrm{Zn}_{\mathrm{i}}$ pairs, similar to what has been observed in $\beta-\mathrm{Zn}_{4} \mathrm{Sb}_{3}$.

\subsection{Minimum Thermal Conductivity}

The low thermal conductivity of the $\mathrm{Zn}_{3} \mathrm{P}_{2}$ film, as well as its weak temperature dependence for the entire temperature range measured, make it interesting for comparison to the minimum thermal conductivity model formulated by Slack ${ }^{27}$ and extended by Cahill and Pohl ${ }^{28}$. The model proposes that the phonon mean free path (MFP) is half the wavelength of a normal mode, $\lambda / 2$, and that the phonon dispersion relationship is that of the Debye model $(\omega=$ $v(2 \pi / \lambda)$, where $v$ is the speed of sound and $\lambda$ is the phonon wavelength). The resulting minimum thermal conductivity is given by

$$
\kappa_{\min }=\left(\frac{\pi}{6}\right)^{1 / 3} k_{B} n^{2 / 3} \sum_{i} v_{i}\left(\frac{T}{\Theta_{i}}\right)^{2} \int_{0}^{\Theta_{i} / T} \frac{x^{3} e^{x}}{\left(e^{x}-1\right)^{2}} d x
$$

where $i$ indicates the polarization ( $l$ and $t$ for the longitudinal and transverse polarizations, respectively) of the three sound modes, $v_{i}$ is the sound velocity, $\mathrm{n}$ is the number density of

atoms, and $\Theta_{i}$ is the Debye temperature given by $\Theta_{i}=v_{i}\left(\hbar / k_{B}\right)\left(6 \pi^{2} n\right)^{1 / 3}$. Equation (10) was 
evaluated using the velocity and atomic density values previously mentioned for use in the Callaway model.

The resulting minimum thermal conductivity curve is shown in Fig. 5 as a blue line with small dashing, where it can be seen that it lies above the thermal conductivity of $\mathrm{Zn}_{3} \mathrm{P}_{2}$ film for all but the lowest temperatures at which measurements were made. The rapid fall off in the minimum thermal conductivity at lower temperatures is expected because of the growing importance of phonons with long MFPs in real solids. The fact that the thermal conductivity of the $\mathrm{Zn}_{3} \mathrm{P}_{2}$ film lies at all below the minimum thermal conductivity, where it is $25 \%$ smaller at room temperature, is peculiar. If the minimum thermal conductivity calculation is extended to $800 \mathrm{~K}$, it has a value of $0.72 \mathrm{~W} / \mathrm{m} \mathrm{K}$ and the thermal conductivity of the samples measured by Brockway et al. ${ }^{20}$ and Nagamoto et al. ${ }^{21}$ would lie above it. This indicates that falling below the minimum is not an intrinsic property of $\mathrm{Zn}_{3} \mathrm{P}_{2}$. Since the formulation of the minimum thermal conductivity concept, there have been few reports on solids (amorphous or crystalline, where in fact crystallinity seems to be a requirement for falling below the "glassy limit" $"$ ) that are found to have thermal conductivities that fall below this limit. Notably, systems that have been identified as such include some layered films, copper based so called "phonon-liquid electroncrystals" (PLECs), and some nanocrystalline (<30 nm crystallites) solids. ${ }^{61-66}$

\subsubsection{Thermal Conductivity Below the Minimum}

As it relates to the present study, the observations of Feser et al. ${ }^{66}$ of thermal conductivities in some CdSe thin films with grain sizes of 3-6 nm falling below the minimum thermal conductivity are particularly interesting. Although the exact cause for the thermal conductivities of their study falling below the minimum was not determined, Feser et al. ${ }^{66}$, based 
on considerations of the phonon dispersion relations of $\mathrm{CdSe}$, were able to reject ballistic phonon transport (often used to explain anomalous reductions in thermal conductivity observed in crystals with boundary dimensions on the order of dominant phonon MFPs ${ }^{67}$ ) as the cause. Furthermore, they reason that the CdSe phonon dispersion relations illustrate the inconsequential nature of thermal transport from optical and short wavelength acoustic phonons. Thus, the minimum thermal conductivity model can overestimate the thermal conductivity of a solid when, for each polarization, a single group velocity is assumed for all modes.

The 40 atom unit cell of $\mathrm{Zn}_{3} \mathrm{P}_{2}$ results in 120 phonon branches ( 3 acoustic and 117 optical) that highly complicate the details of thermal transport. To the best of the authors' knowledge, there is at present no publication of the phonon dispersion relations for $\mathrm{Zn}_{3} \mathrm{P}_{2}$. The unavailability of the phonon dispersion relations makes it difficult to elucidate the exact cause for the thermal conductivity of the nanocrystalline $\mathrm{Zn}_{3} \mathrm{P}_{2}$ film falling below the "glassy limit", and highlights the need for such a study. Given that low thermal conductivity has been observed in every report on $\mathrm{Zn}_{3} \mathrm{P}_{2}$ and its family of isomorphic compounds, it is likely that a relatively short MFP for the dominant heat carrying phonons is an intrinsic property of the crystal structure. Furthermore, that the effect of nanostructuring produced no reduction in the room temperature thermal conductivity of the nanowire pellets in the study of Brockway et al. ${ }^{20}$ suggests that these boundary effects occur at length scales smaller than 30-50 nm. If the largest room temperature thermal conductivity reported for $\mathrm{Zn}_{3} \mathrm{P}_{2}\left(\sim 2.5 \mathrm{~W} / \mathrm{m} \mathrm{K}\right.$ by Masumoto et al. $\left.{ }^{15}\right)$ is used as an estimate for the bulk value, the $\mathrm{Zn}_{3} \mathrm{P}_{2}$ film of the present study has the value $\kappa_{\text {Film }}=$ $0.2 \kappa_{B u l k}$ at room temperature. If it is assumed that the phonon MFP is independent of frequency and that the scattering processes of the film are equivalent to that of the bulk, with the effect of additional boundary scattering given by Matthiessen's rule, we find that 


$$
\frac{\kappa_{\text {Film }}}{\kappa_{\text {Bulk }}}=\frac{L_{\text {Film }}}{L_{\text {Film }}+L_{\text {Bulk }}}
$$

where $\kappa_{\text {Film }}$ and $\kappa_{\text {Bulk }}$ are the thermal conductivities of the film and bulk, respectively, and $L_{\text {Film }}$ and $L_{B u l k}$ are the MFP due to the additional boundaries from nanostructuring in the film and the MFP of bulk, respectively. If the largest crystallite size $(6.1 \mathrm{~nm})$ determined in this study is used for $L_{\text {Film }}$, we find that the MFP of bulk $\mathrm{Zn}_{3} \mathrm{P}_{2}$ is $24.4 \mathrm{~nm}$. From this result, the nanowires of Brockway et al. ${ }^{20}$ would be too large to reduce this MFP, but the $<7 \mathrm{~nm}$ crystallites in the film of the present study would greatly reduce it.

\section{Conclusion}

A nanocrystalline $\mathrm{Zn}_{3} \mathrm{P}_{2}$ thin film was fabricated using PLD and crystallized by RTA at $773 \mathrm{~K}$ for $120 \mathrm{~s}$. XRD analysis and HRTEM indicated that the film was polycrystalline with an average crystallite size of $4.1 \mathrm{~nm}$. The thermal conductivity of the $\mathrm{Zn}_{3} \mathrm{P}_{2}$ film was measured from $80 \mathrm{~K}$ to $294 \mathrm{~K}$ using the $3 \omega$ method. The thermal conductivity in this range showed weak temperature dependence and at $294 \mathrm{~K}$ had its highest value of $0.49 \mathrm{~W} / \mathrm{m} \mathrm{K}$. The thermal conductivity data were well fit with the Callaway model, where only boundary and point defect phonon scattering processes were initially needed to achieve a good fit. The boundary scattering term was in good agreement with the average crystallite size. From considerations of the formation energies and site availabilities, the point defect scattering is believed to have its origins in $23 \%$ of Zn sites being occupied by vacancies, with a commensurate number of interstitial sites being occupied by $\mathrm{Zn}$ atoms. The inclusion of resonant phonon scattering processes in the Callaway model, to account for phonon scattering by Zn interstitials occupying lattice voids, also resulted in a good fit to the data. From previously reported results on structure refinement in $\mathrm{Zn}_{3} \mathrm{P}_{2}$, it is suggested 
that the formation of a high number of $\mathrm{V}_{\mathrm{Zn}}-\mathrm{Zn}_{\mathrm{i}}$ pairs is made possible by the $\mathrm{Zn}(2)$ atom having an equal probability of occupying a lattice site or void. The thermal conductivity of the $\mathrm{Zn}_{3} \mathrm{P}_{2}$ film fell below the minimum thermal conductivity for all but the lowest temperatures measured. The unavailability of a phonon dispersion for $\mathrm{Zn}_{3} \mathrm{P}_{2}$ makes elucidation of the thermal conduction that arises from its 120 phonon branches difficult. With such remarkably low thermal conductivity not having been observed in previous reports on $\mathrm{Zn}_{3} \mathrm{P}_{2}$, it is believed that the findings of present study result from the $<7 \mathrm{~nm}$ crystallite sizes of the $\mathrm{Zn}_{3} \mathrm{P}_{2}$ film.

As a number of authors have previously reported that there is no appreciable change in the electrical conductivity of $\mathrm{Zn}_{3} \mathrm{P}_{2}$ in the presence of grain boundaries ${ }^{22,25}$, the reductions in thermal conductivity achieved through nanostructuring in this study is promising for increasing the viability of $\mathrm{Zn}_{3} \mathrm{P}_{2}$ 's use in thermoelectric applications. As future work may involve the tailoring of point defects to achieve better electronic conduction properties for improved thermoelectric performance, we note that $\mathrm{Zn}$ interstitials were found to have only a small effect on the thermal conductivity and as this defect type is believed to act as an electron donor ${ }^{38}$, it is suggested that $\mathrm{Zn}$ deficient compositions should be desirable for an increase in p-type conductivity while having a negligible effect on the thermal properties.

\section{Acknowledgments}

Portions of this work were funded by the Office of Naval Research; grant number N00014-11-1-0658.

\section{$\underline{\text { References }}$}


(1) Lee, S.-M.; Cahill, D. G.; Venkatasubramanian, R. Thermal Conductivity of Si-Ge Superlattices. Appl. Phys. Lett. 1997, 70 (22), 2957.

(2) Venkatasubramanian, R. Lattice Thermal Conductivity Reduction and Phonon Localizationlike Behavior in Superlattice Structures. Phys. Rev. B 2000, 61 (4), 3091-3097.

(3) Boukai, A. I.; Bunimovich, Y.; Tahir-Kheli, J.; Yu, J.-K.; Goddard, W. a; Heath, J. R. Silicon Nanowires as Efficient Thermoelectric Materials. Nature 2008, 451 (7175), 168-171.

(4) Hochbaum, A. I.; Chen, R.; Delgado, R. D.; Liang, W.; Garnett, E. C.; Najarian, M.; Majumdar, A.; Yang, P. Enhanced Thermoelectric Performance of Rough Silicon Nanowires. Nature 2008, 451 (7175), 163-167.

(5) Nolas, G.; Cohn, J.; Slack, G. Effect of Partial Void Filling on the Lattice Thermal Conductivity of Skutterudites. Phys. Rev. B 1998, 58 (1), 164-170.

(6) Nolas, G. S.; Slack, G. a.; Morelli, D. T.; Tritt, T. M.; Ehrlich, a. C. The Effect of Rare-Earth Filling on the Lattice Thermal Conductivity of Skutterudites. J. Appl. Phys. 1996, 79 (8), 4002.

(7) Cohn, J. L.; Nolas, G. S.; Fessatidis, V.; Metcalf, T. H.; Slack, G. a. Glass-Like Heat Conduction in High-Mobility Crystalline Semiconductors. Phys. Rev. Lett. 1998, 82 (4), 779-782.

(8) Dames, C.; Chen, G. Thermal Conductivity of Nanostructured Thermoelectric Materials. Thermoelectr. Handb. Macro to Nano 2006, No. 42, 1-16.

(9) Snyder, G. J.; Toberer, E. S. Complex Thermoelectric Materials. Nat. Mater. 2008, 7 (2), 105114.

(10) Wyeth, N. C.; Catalano, A. Spectral Response Measurements of Minority-Carrier Diffusion Length in Zn3P2. J. Appl. Phys. 1979, 50 (3), 1403-1407.

(11) Wadia, C.; Alivisatos, a. P.; Kammen, D. M. Materials Availability Expands the Opportunity for Large-Scale Photovoltaics Deployment. Environ. Sci. Technol. 2009, 43 (6), 2072-2077.

(12) Fagen, E. a. Optical Properties of Zn3P2. J. Appl. Phys. 1979, 50 (10), 6505-6515.

(13) Spitzer, D. P.; Castellion, G. a.; Haacke, G. Anomalous Thermal Conductivity of Cd3As2 and the Cd3As2-Zn3As2 Alloys. J. Appl. Phys. 1966, 37 (10), 3795.

(14) Armitage, D.; Goldsmid, H. J. The Thermal Conductivity of Cadmium Arsenide. J. Phys. C Solid State Phys. 2002, 2 (11), 2138-2145.

(15) Masumoto, K.; Isomura, S. Physical and Electronic Properties of Semiconducting Solid Solutions of the Cd3P2-Zn3P2 System. Phys. Status Solidi 1971, 6 (2), 515-523.

(16) Misiewicz, J. Inter-Band Transitions in Zn3P2. J. Phys. Condens. Matter 1990, 2 (8), 2053-2072.

(17) Zanin, I. E.; Aleinikova, K. B.; Antipin, M. Y.; Afanas'ev, M. M. Analysis of Chemical Bonding in Zn3P2 Crystals from X-Ray Diffraction Data. Crystallogr. Reports 2004, 49 (4), 579-584. 
(18) Spitzer, D. P. Lattice Thermal Conductivity of Semiconductors: A Chemical Bond Approach. J. Phys. Chem. Solids 1970, 31 (1), 19-40.

(19) Roufosse, M.; Klemens, P. Thermal Conductivity of Complex Dielectric Crystals. Phys. Rev. B 1973, 7 (12), 5379-5386.

(20) Brockway, L.; Vasiraju, V.; Asayesh-Ardakani, H.; Shahbazian-Yassar, R.; Vaddiraju, S. Thermoelectric Properties of Large-Scale Zn3P2 Nanowire Assemblies. Nanotechnology 2014, 25 (14), 145401.

(21) Nagamoto, Y.; Hino, K.; Yoshitake, H.; Koyanagi, T. Thermoelectric Properties of a-Zn3P2. 17th Int. Conf. Thermoelectr. 1998, 354-357.

(22) Bhushan, M.; Catalano, A. Polycrystalline Zn3P2 Schottky Barrier Solar Cells. Appl. Phys. Lett. 1981, $38(1), 39-41$.

(23) Luber, E. J.; Mobarok, M. H.; Buriak, J. M. Solution-Processed Zinc Phosphide (a-Zn3P2) Colloidal Semiconducting Nanocrystals for Thin Film Photovoltaic Applications. ACS Nano 2013, 7 (9), 8136-8146.

(24) Kimball, G. M.; Müller, A. M.; Lewis, N. S.; Atwater, H. a. Photoluminescence-Based Measurements of the Energy Gap and Diffusion Length of Zn3P2. Appl. Phys. Lett. 2009, 95 (11), 3-6.

(25) Babu, V. S.; Vaya, P. R.; Sobhanadri, J. Electrical and Thermoelectrical Properties of Zn3P2 Films Grown by the Hot Wall Epitaxy Technique. J. Appl. Phys. 1988, 64 (4), 1922-1926.

(26) Cahill, D. G. Thermal Conductivity Measurement from 30 to $750 \mathrm{~K}$ : The $3 \omega$ Method. Rev. Sci. Instrum. 1990, 61 (2), 802.

(27) Slack, G. A. The Thermal Conductivity of Nonmetallic Crystals. Solid State Phys. - Adv. Res. Appl. 1979, 34 (C), 1-71.

(28) Cahill, D. G.; Pohl, R. O. Heat Flow and Lattice Vibrations in Glasses. Solid State Commun. 1989, 70 (10), 927-930.

(29) Cahill, D. G.; Katiyar, M.; Abelson, J. R. Thermal Conductivity of a-Si:H Thin Films. Phys. Rev. B 1994, 50 (9), 6077-6082.

(30) University., P.; Center., T. P. R.; Touloukian, Y. S. Thermophysical Properties of Matter - The TPRC Data Series; IFI/Plenum: New York, 2003.

(31) Misiewicz, J. Optical Vibrations in the Zn3P2 Lattice. J. Phys. Condens. Matter 1989, 1, 92839299.

(32) Scherrer, P. Bestimmung Der Grösse Und Der Inneren Struktur von Kolloidteilchen Mittels Röntgenstrahlen. Nachrichten von der Gesellschaft der Wissenschaften zu Göttingen, Math. Klasse 1918, 1918, 98-100. 
(33) Langford, J. I.; Wilson, A. J. C. Seherrer after Sixty Years: A Survey and Some New Results in the Determination of Crystallite Size. J. Appl. Cryst. 1978, 11, 102-113.

(34) Cahill, D. G.; Watson, S. K.; Pohl, R. O. Lower Limit to the Thermal Conductivity of Disordered Crystals. Phys. Rev. B 1992, 46 (10), 6131-6140.

(35) Callaway, J. Model for Lattice Thermal Conductivity at Low Temperatures. Phys. Rev. 1959, 113 (4), 1046-1051.

(36) Freyland, W.; Goltzene, A.; Grosse, P.; Harbeke, G.; Lehmann, H.; Madelung, O.; Richter, W.; Schwab, C.; Weiser, G.; Werheit, H.; et al. Physics of Non-Tetrahedrally Bonded Elements and Binary Compounds I, 1st ed.; Madelung, C., Ed.; Springer-Verlag Berlin Heidelberg, 1983.

(37) Parrott, J. E. The Thermal Conductivity of Sintered Semiconductor Alloys. J. Phys. C Solid State Phys. 1969, 2 (1), 147-151.

(38) Demers, S.; Van De Walle, A. Intrinsic Defects and Dopability of Zinc Phosphide. Phys. Rev. B 2012, 85 (19), 1-10.

(39) Catalano, A.; Hall, R. B. Defect Dominated Conductivity in Zn3P2. J. Phys. Chem. Solids 1980, $41,635-640$.

(40) Klemens, P. G. The Scattering of Low-Frequency Lattice Waves by Static Imperfections. Proc. Phys. Soc. Sect. A 2002, 68 (12), 1113-1128.

(41) Slack, G. A. Thermal Conductivity of MgO, Al2O3, MgAl2O4, and Fe2O4 Crystals from 3 to 300 K. Phys. Rev. 1962, 126 (2), 427-441.

(42) O'Hara, S. G.; Sellers, G. J.; Anderson, A. C. Influence of Hydrogen on the Thermal Conductivities of Superconducting Nb and Ta. Phys. Rev. B 1974, 10 (7), 2777-2781.

(43) Nolas, G. S.; Cohn, J. L.; Slack, G. a.; Schujman, S. B. Semiconducting Ge Clathrates: Promising Candidates for Thermoelectric Applications. Appl. Phys. Lett. 1998, 73 (2), 178-180.

(44) Morelli, D. T.; Meisner, G. P. Low Temperature Properties of the Filled Skutterudite CeFe4Sb12. J. Appl. Phys. 1995, 77 (8), 3777-3781.

(45) Sales, B. C.; Mandrus, D.; Williams, R. K. Filled Skutterudite Antimonides: A New Class of Thermoelectric Materials. Science (80-. ). 1996, 272 (5266), 1325-1328.

(46) Keppens, V.; Mandrus, D.; Sales, B. C.; Chakoumakos, B. C.; Dai, P.; Coldea, R.; Maple, M. B.; Gajewski, D. A.; Freeman, E. J.; Bennington, S. Localized Vibrational Modes in Metallic Solids. Lett. to Nat. 1998, 395 (October), 876-878.

(47) Long, G. J.; Hermann, R. P.; Grandjean, F.; Alp, E. E.; Sturhahn, W.; Johnson, C. E.; Brown, D. E.; Leupold, O.; Rüffer, R. Strongly Decoupled Europium and Iron Vibrational Modes in Filled Skutterudites. Phys. Rev. B 2005, 71 (14), 140302. 
(48) Pohl, R. O. Thermal Conductivity and Phonon Resonance Scattering. Phys. Rev. Lett. 1962, 8 (12), 481-483.

(49) Tse, J. S.; White, M. A. Origin of Glassy Crystalline Behavior in the Thermal Properties of Clathrate Hydrates: A Thermal Conductivity Study of Tetrahydrofuran Hydrate. J. Phys. Chem. 1988, 92 (17), 5006-5011.

(50) Yang, J.; Morelli, D.; Meisner, G.; Chen, W.; Dyck, J.; Uher, C. Effect of Sn Substituting for Sb on the Low-Temperature Transport Properties of Ytterbium-Filled Skutterudites. Phys. Rev. B 2003, 67 (16), 1-6.

(51) Nolas, G. S.; Fowler, G.; Yang, J. Assessing the Role of Filler Atoms on the Thermal Conductivity of Filled Skutterudites. J. Appl. Phys. 2006, 100 (4), 043705.

(52) Klitsner, T.; Pohl, R. Phonon Scattering at Silicon Crystal Surfaces. Phys. Rev. B 1987, 36 (12), $6551-6565$.

(53) Slack, G. A.; Rowe, D. M. CRC Handbook of Thermoelectrics. New York 1995, 16 (1-4).

(54) Caillat, T.; Fleurial, J.-P.; Borshchevsky, A. Preparation and Thermoelectric Properties of Semiconducting Zn4Sb3. J. Phys. Chem. Solids 1997, 58 (7), 1119-1125.

(55) Snyder, G. J.; Christensen, M.; Nishibori, E.; Caillat, T.; Iversen, B. B. Disordered Zinc in Zn4Sb3 with Phonon-Glass and Electron-Crystal Thermoelectric Properties. Nat. Mater. 2004, 3 (7), 458463.

(56) Zhang, L. .; Tsutsui, M.; Ito, K.; Yamaguchi, M. Thermoelectric Properties of Zn4Sb3 Thin Films Prepared by Magnetron Sputtering. Thin Solid Films 2003, 443 (1-2), 84-90.

(57) Toberer, E. S.; Sasaki, K. a.; Chisholm, C. R. I.; Haile, S. M.; Goddard, W. a.; Snyder, G. J. Local Structure of Interstitial Zn in B-Zn4Sb3. Phys. Status Solidi - Rapid Res. Lett. 2007, 1 (6), $253-$ 255.

(58) Cargnoni, F.; Nishibori, E.; Rabiller, P.; Bertini, L.; Snyder, G. J.; Christensen, M.; Gatti, C.; Iversen, B. B. Interstitial Zn Atoms Do the Trick in Thermoelectric Zinc Antimonide, Zn4Sb3: A Combined Maximum Entropy Method X-Ray Electron Density and Ab Initio Electronic Structure Study. Chem. - A Eur. J. 2004, 10 (16), 3861-3870.

(59) Lin, J.; Li, X.; Qiao, G.; Wang, Z.; Carrete, J.; Ren, Y.; Ma, L.; Fei, Y.; Yang, B.; Lei, L.; et al. Unexpected High-Temperature Stability of B-Zn 4 Sb 3 Opens the Door to Enhanced Thermoelectric Performance. J. Am. Chem. Soc. 2014, 136 (4), 1497-1504.

(60) Zanin, I. E.; Aleinikova, K. B.; Afanasiev, M. M.; Antipin, M. Y. Structure of Zn3P2. J. Struct. Chem. 2004, 45 (5), 844-848.

(61) Chiritescu, C.; Cahill, D. G.; Nguyen, N.; Johnson, D.; Bodapati, A.; Keblinski, P.; Zschack, P. Ultralow Thermal Conductivity in Disordered, Layered WSe2 Crystals. Science 2007, 315 (5810), 351-353. 
(62) Foley, B. M.; Brown-Shaklee, H. J.; Campion, M. J.; Medlin, D. L.; Clem, P. G.; Ihlefeld, J. F.; Hopkins, P. E. Glass-Like Thermal Conductivity of (010)-Textured Lanthanum-Doped Strontium Niobate Synthesized with Wet Chemical Deposition. J. Am. Ceram. Soc. 2015, 98 (2), 624-628.

(63) He, Y.; Day, T.; Zhang, T.; Liu, H.; Shi, X.; Chen, L.; Snyder, G. J. High Thermoelectric Performance in Non-Toxic Earth-Abundant Copper Sulfide. Adv. Mater. 2014, 26 (23), 39743978.

(64) Weldert, K. S.; Zeier, W. G.; Day, T. W.; Panthöfer, M.; Snyder, G. J.; Tremel, W. Thermoelectric Transport in Cu7PSe6 with High Copper Ionic Mobility. J. Am. Chem. Soc. 2014, 136 (34), 12035-12040.

(65) Buscaglia, M. T.; Maglia, F.; Anselmi-Tamburini, U.; Marré, D.; Pallecchi, I.; Ianculescu, A.; Canu, G.; Viviani, M.; Fabrizio, M.; Buscaglia, V. Effect of Nanostructure on the Thermal Conductivity of La-Doped SrTiO3 Ceramics. J. Eur. Ceram. Soc. 2014, 34 (2), 307-316.

(66) Feser, J. P.; Chan, E. M.; Majumdar, A.; Segalman, R. a.; Urban, J. J. Ultralow Thermal Conductivity in Polycrystalline CdSe Thin Films with Controlled Grain Size. Nano Lett. 2013, 13 (5), 2122-2127.

(67) Wang, Z.; Alaniz, J. E.; Jang, W.; Garay, J. E.; Dames, C. Thermal Conductivity of Nanocrystalline Silicon: Importance of Grain Size and Frequency-Dependent Mean Free Paths. Nano Lett. 2011, 11 (6), 2206-2213. 

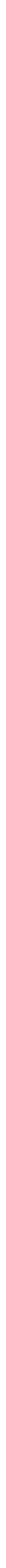